\title{
Unsupervised Identification of Isotope-Labeled Peptides
}

Joshua E Goldford ${ }^{13}$ and Igor GL Libourel ${ }^{124}$

${ }^{1}$ Biotechnology institute, University of Minnesota, Saint Paul, MN 55108

${ }^{2}$ Department of Plant Biology, 1500 Gortner Avenue, University of Minnesota, Saint Paul, MN 55108.

${ }^{3}$ Current address: Bioinformatics Program, Boston University, Boston, MA 02215

${ }^{4}$ Corresponding author

Email IGLL: Libourel@,umn.edu

\section{Table of Contents}

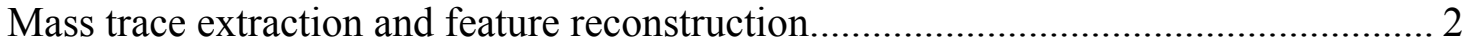

Feature identification and discrimination ......................................................... 2

Identification of unlabeled peptides............................................................. 2

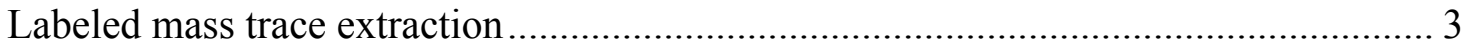

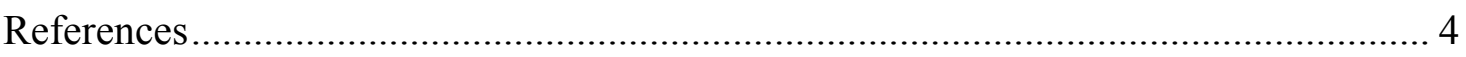

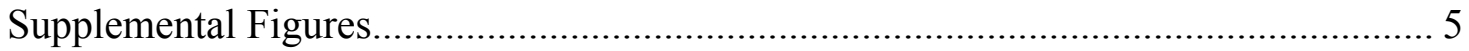

Figure S1. Manually extracted features of eighteen isotope-labeled peptides from

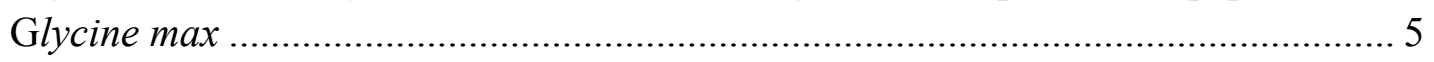

Figure S2: Processing of raw mass spectra from isotopically labeled samples ......... 6

Figure S3: Integration of mass deviation and intensity correlation .......................... 6

Figure S4: Feature reconstruction of $7 \% \mathrm{U}^{13} \mathrm{C}$ labeled $E$. coli peptides. ................. 7

Figure S5: Amino acid mass distributions (AMDs) determined from the peptides of the soy $\beta$-Conglicinin protein (Fig 6 main manuscript) ................................... 8 


\section{Mass trace extraction and feature reconstruction}

Raw orbitrap data (mzXML format) was converted to the mzML format in the TOPPAS environment ${ }^{1}$, and OpenMS functions were used for subsequent analysis ${ }^{2}$. LC mass peaks were identified using the PeakPickerHiRes algorithm, and were converted into $\mathrm{m} / \mathrm{z}$ traces with the Mass Trace Extractor algorithm using an $\mathrm{S} / \mathrm{N}$ cutoff of 1 . Custom software was written to extract intensity, noise and $\mathrm{m} / \mathrm{z}$ data from profile scans using the MSFileReader API (Thermo Fisher Scientific, San Jose CA). All software was written in $\mathrm{C}++$, and compiled into a MATLAB (MathWorks, Natick MA) executable using the mex function.

To filter $\mathrm{m} / \mathrm{z}$ traces for feature reconstruction, an $\mathrm{S} / \mathrm{N}$-weighted $\mathrm{m} / \mathrm{z}$ value was determined for each $\mathrm{m} / \mathrm{z}$ trace, and for all $\mathrm{m} / z$ values in a mass trace, $m_{i}$, the isotopic accuracy, $d_{i}$, was calculated by taking the signed difference from the closest simulated isotopologue for a target peptide, $m_{k, \text { sim }}$ such that $d_{i}=\frac{m_{i}-m_{k, s i m}}{m_{k, s i m}} \times 10^{6}$. The isotopic precision, $\Delta_{i, j}$, between mass trace $i$ and $j$ was calculated by taking the absolute difference between respective isotopic accuracies, where $\Delta_{i, j}=\left|d_{i}-d_{j}\right|$. The isotopic precision was then mapped to the domain $0 \ldots 1$, using a gaussian transformation: $w_{i, j}=e^{\frac{-\left(\Delta_{i, j}\right)^{2}}{2 \sigma^{2}}}$ where $\sigma$ is the mean deviation in ppm one would expect between traces. The function was parameterized with observed isotopic deviations within $\mathrm{m} / \mathrm{z}$ traces.

\section{Feature identification and discrimination}

MaxQuant v 1.3.0.534 was used for automatic peptide identification in natural abundant reference samples. Parameters were left to default unless specified otherwise. Identification was performed iteratively by first identifying which proteins were implicated by at least one peptide, using the full proteome for Glysine max (3847, UniProt Consortium). A subsequent search was restricted to peptides of proteins implicated by the first search. The proteome database was loaded into Andromeda and allowed modifications included carbamidomethylation of cysteine, oxidation of methionine, and deamination of glutamine and asparagine. The digestion of trypsin or chymotrypsin was specified with a multiplicity of 1 , and a mass accuracy for the first and second peptide searches was set to 20 and 6ppm, respectively. Peptide false discovery rate was set to 0.01 . The same procedure was followed for Escherichia coli datasets (511145, UniProt Consortium).

\section{Identification of unlabeled peptides}

Paired ${ }^{13} \mathrm{C}$-labeled and unlabeled experiments were performed on E. coli and G. $\max$ to obtain labeled and unlabeled protein samples that were treated identically in every aspect. Tryptic and chymotryptic digests of paired samples were run consecutively on an orbital trap mass spectrometer (orbitrap). Unlabeled samples were used for peptide identification, and for the determination of reference values for retention times and signal intensities. Peptides in the unlabeled sample were analyzed through fragmentation and 
identified using MaxQuant, which made use of the genomic sequence to determine the uniqueness of each identified peptide. In a first pass, G. max and E. coli samples were analyzed for the occurrence of peptides from proteins specific to the investigated organism and common contaminants. In a second pass, the peptide database was restricted to proteins that were implicated during the first pass, to find peptides that were unique within this reduced database but not necessarily unique within the complete proteome. This iterative approach resulted in the recovery $30-50 \%$ additional peptides.

\section{Labeled mass trace extraction}

A total of eighteen labeled peptides derived from the soy storage protein $\beta$-conglycinin were manually annotated and used as training set (Supporting Fig S1). This training set was previously used for flux analysis ${ }^{3}$, and contained peptides of lengths between 3 and 12 amino acids, spanned a range of absolute intensities from $1.2 \cdot 10^{5}$ to $3.6 \cdot 10^{8}$, and included seventeen out of twenty amino acids. The set was first used to investigate the parameter settings for $m / z$ trace extraction using OpenMS. To optimize $\mathrm{m} / \mathrm{z}$ trace extraction, OpenMS parameters values were adjusted to maximize the similarity between any pair of $\mathrm{m} / \mathrm{z}$ traces within an isotopic cluster of the peptides in this training set using a grid search. For a pair of $\mathrm{m} / \mathrm{z}$ traces (A, B) within an isotopic cluster, the similarity was captured by determining the degree of overlap in time using the Jaccard similarity index: $j_{(A, B)}=\frac{A \cap B}{A \cup B}$. Within the context of LC-MS data, $j$ measures the number of shared scans between two $\mathrm{m} / \mathrm{z}$ traces as a fraction of number of total scan events for both $\mathrm{m} / \mathrm{z}$ traces. The index was used to tune the optimal sample rate and mass precision constraints. Note that too permissive a mass consistency requirement, or too permissive a sample rate constraint, may cause erroneously conjoined $\mathrm{m} / \mathrm{z}$ traces, which artificially elongates $\mathrm{m} / \mathrm{z}$ traces and lowers $j$. Conversely, too stringent a sample rate or mass consistency setting causes truncation of $\mathrm{m} / \mathrm{z}$ traces, again lowering $j$. The Jaccard indices of all trace comparisons were summed over all peptides within the training set yielding an aggregate statistic, $J$. A sample rate of 0.5 and mass consistency of $4 \mathrm{ppm}$ resulted in the maximum $J$ if the inclusion of all manually identified $\mathrm{m} / \mathrm{z}$ traces in the training set was required. The 372 extracted $\mathrm{m} / \mathrm{z}$ traces for the training set included a total of 34,564 individual measurements. The range of $m / z$ trace lengths was between $0.82-838.19$ seconds, with a mean of 77.65 and median of 27.54 seconds. The minimal extracted intensity value was 524, and the minimum $\mathrm{S} / \mathrm{N}$ ratio was 1.06. The full-width-half-max (FWHM) of traces had a mean width of 9.70 seconds (SD 9.21s) with a full range between 0.15 and $63.5 \mathrm{~s}$. 
Goldford and Libourel: Unsupervised Identification of Isotope-Labeled Peptides

\section{References}

(1) Junker, J.; Bielow, C.; Bertsch, A.; Sturm, M.; Reinert, K.; Kohlbacher, O. J. Proteome Res. 2012, 11, 3914-3920.

(2) Reinert, K.; Kohlbacher, O. Methods Mol. Biol. 2010, 604, 201-211.

(3) Mandy, D. E.; Goldford, J. E.; Yang, H.; Allen, D. K.; Libourel, I. G. L. Plant J. 2014, 77, 476-486. 


\section{Supplemental Figures}

$\mathrm{LLK} ;+2$

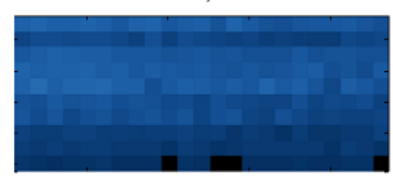

NKNPFHFNSK;+4

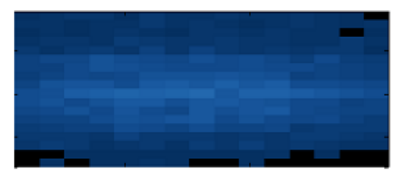

SRDPIYSNK;+3

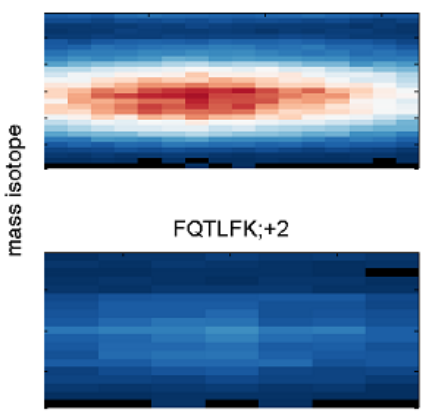

DIENLIK;+2



FEEINKVLFGR;+3


LQESVIVEISKK;+3
DYR +2

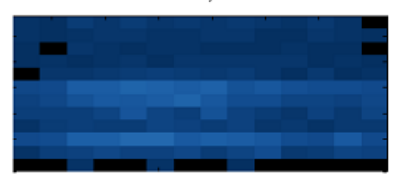

VLFSR;+2

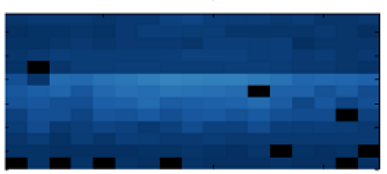

NFLAGSK;+2

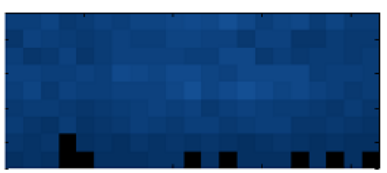

FETLFK;+2

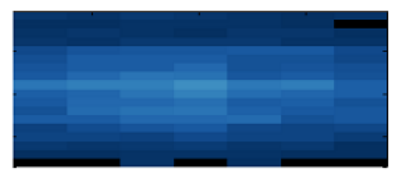

LQSGDALR;+2
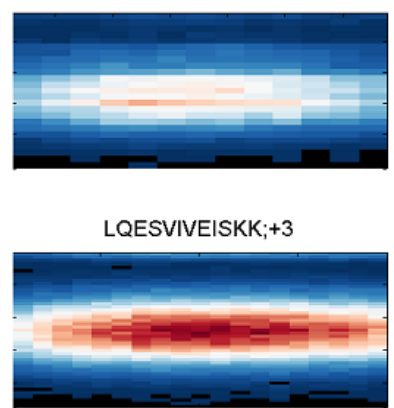

600

1
800

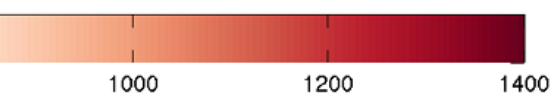

Figure S1. Manually extracted features of eighteen isotope-labeled peptides from Glycine max

Features are shown as heatmaps plotted as function of the retention time (x-axis) and $\mathrm{m} / \mathrm{z}$ (y-axis). The estimated ion counts are false colored from blue to red, and black represents instances of no observed signal. 

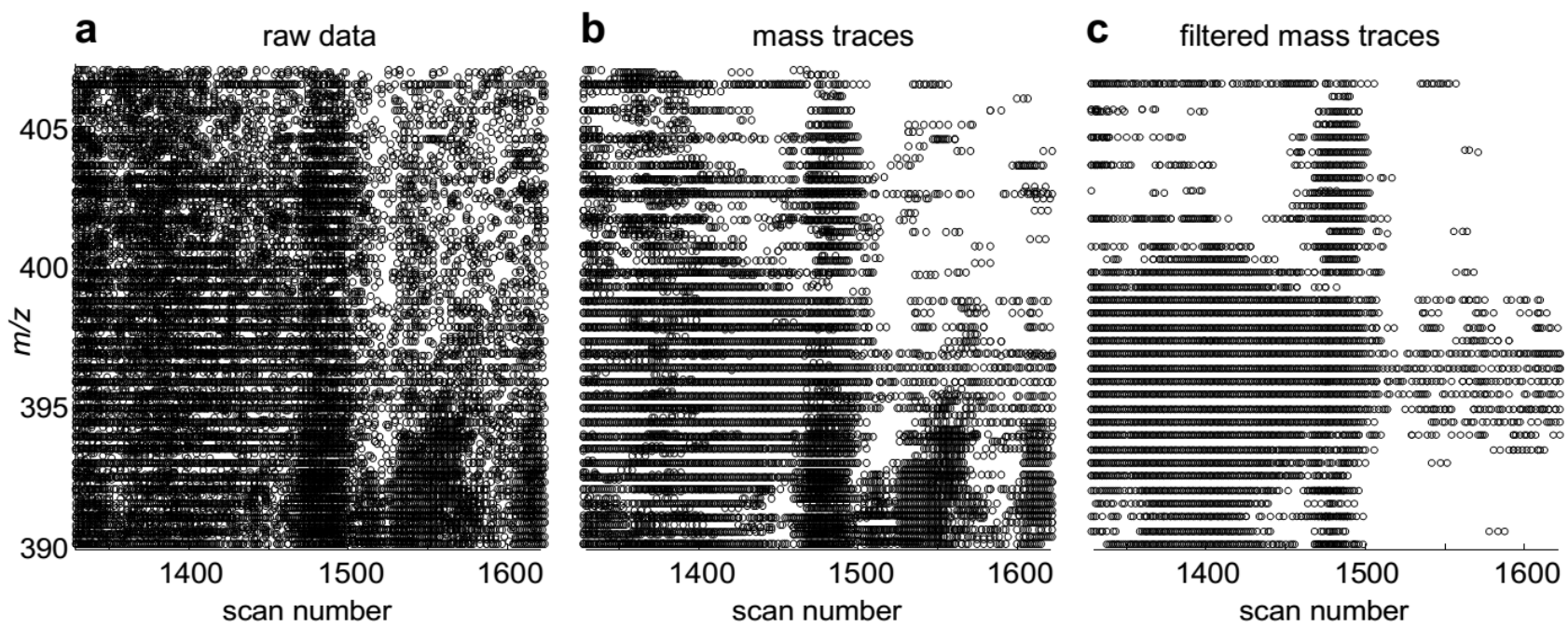

Figure S2: Processing of raw mass spectra from isotopically labeled samples

Raw mass spectra of labeled samples (a) were preprocessed with OpenMS, which grouped measurements into $\mathrm{m} / \mathrm{z}$ traces (b). $M / z$ traces were further filtered using a mass accuracy cutoff (MAC) and a retention time window (RTW), which were calculated from reference masses and retention times of naturally abundant peptides, respectively.
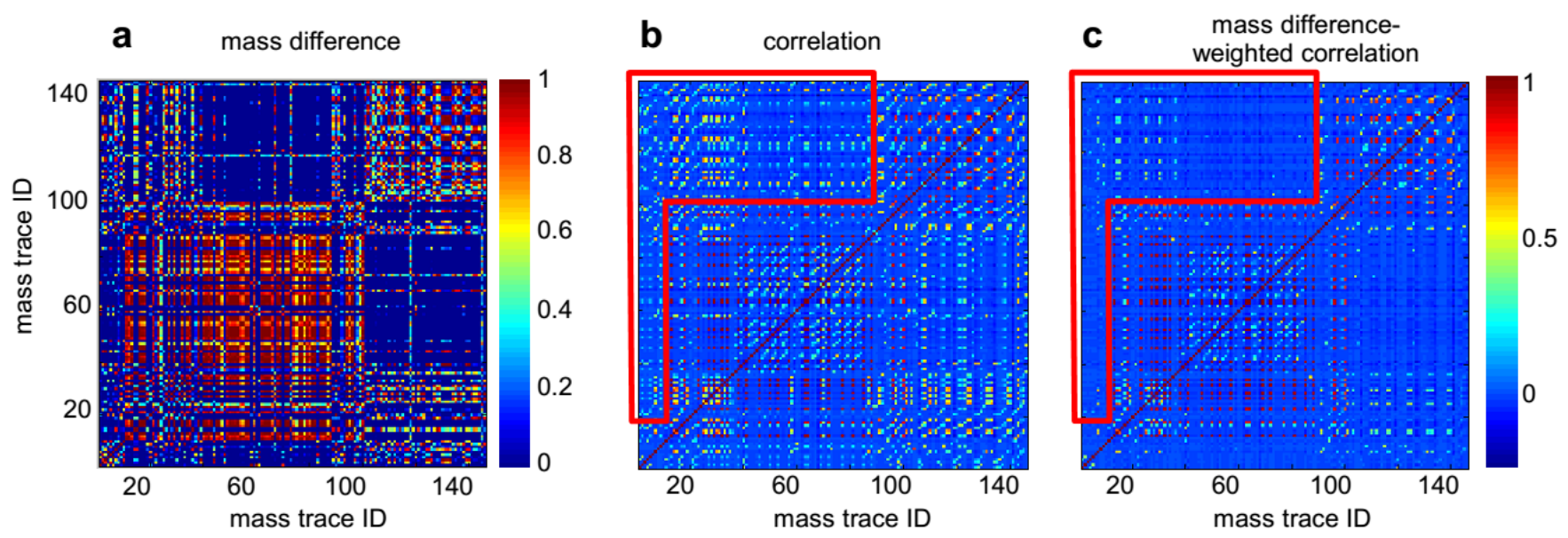

Figure S3: Integration of mass deviation and intensity correlation

Features were identified by integrating isotopic precision (a) and ion chromatogram correlations (b). A mass difference weighted correlation matrix was constructed via element-wise multiplication. Within the red demarcation many of the intensity correlated signals disappeared from the mass-weighted correlation, as the mass differences of these traces did not correlate. 

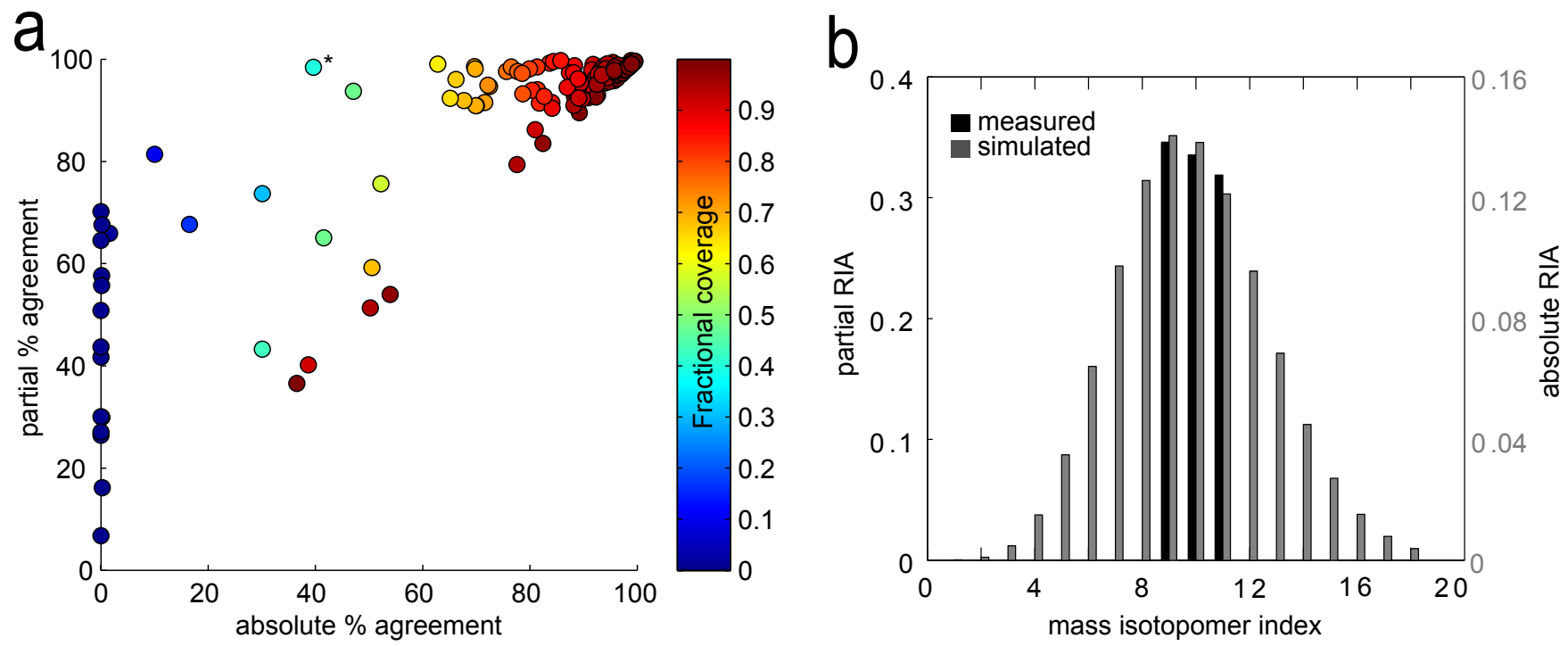

Figure S4: Feature reconstruction of $7 \% \mathrm{U}_{-}{ }^{13} \mathrm{C}$ labeled $E$. coli peptides.

For the set of peptides with unique features at a MAC of $+/-5 \mathrm{ppm}$ and an RTW of $+/-1$ minute, absolute (x-axis) and relative (y-axis) \% agreement (PA) were calculated (a). The PA was calculated by taking the partial $L_{1}$-norm of the difference between simulated and measured peptide isotopes. Absolute PA was determined by taking the difference between all simulated and measured isotopes, and partial PA was determined by taking the difference between calculated and optimally rescaled, measured relative isotopologue abundances (RIA). The color scale represents the fractional coverage of the observed signal relative to the complete simulated distribution. In many cases percent agreement could be improved substantially by using PA. If features moved into the $>90 \%$ agreement they were likely incompletely reconstructed correct features. However, several features did not benefit from rescaling, suggesting that these were not the correct features for the target peptides. An example peptide (* data point in (a)) between a simulated PMD (grey bars) and measured PMD (black bars) showing a likely incomplete feature (b). Most features that showed $<10$ percent agreement improved drastically using the PA comparison, but never increased to $>70 \mathrm{PA}$, suggesting that these were all incorrect features. Regardless, the very low coverage of the PMDs already suggested that these features were incorrect. 


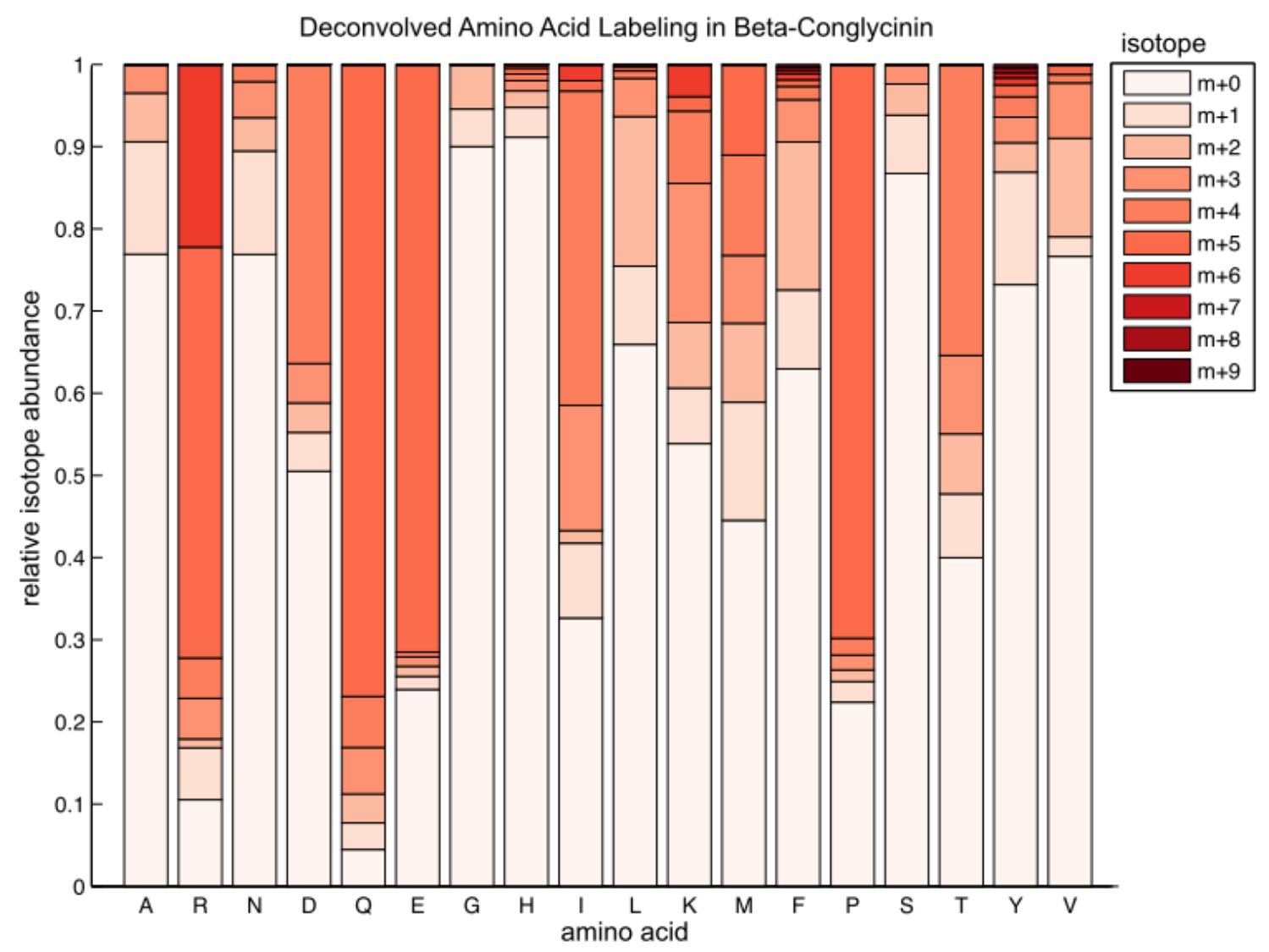

Figure S5: Amino acid mass distributions (AMDs) determined from the peptides of the soy $\beta$-Conglicinin protein (Fig 6 main manuscript).

Isotopomers are intensity coded. The AMDs were fitted using custom made software (https://github.com/LibourelLab/IsotopeLabeledPeptideCode) to the identified peptide mass distributions (PMDs). Notice that glutamine (Q), which was supplied as the uniformly labeled 13C source, was as expected determined to be highly labeled. Glutamate (E) was also determined to be highly uniformly labeled, suggesting that most glutamate was derived from glutamine. Conversely, asparagine, which was supplied as an unlabeled amino acid, was determined to be essentially unlabeled by the deconvolution. 